\title{
Waugh's Syndrome: Report of Two Children with Intussusception
}

\begin{abstract}
Yousuf Aziz Khan ${ }^{1}$ Sunil Kumar Yadav ${ }^{1}$ Ashraf Elkholy ${ }^{2,3}$
${ }^{1}$ Department of Paediatric Surgery, Ibn Sina Specialized Surgical Hospital, Al-Sabah Health Region, Safat, Kuwait, Kuwait, Kuwait

2 Department of Paediatric Surgery, Ibn Sina Specialized Surgical Hospital, Ibn Sina Hospital Ministry of Health, Kuwait, Kuwait, Kuwait

${ }^{3}$ Department of Pediatric Surgery, Kasr Elaini School of Medicine Cairo University, Kasr Elainy Faculty of Medicine, Cairo, Cairo, Egypt

Address for correspondence Yousuf Aziz Khan, MBBS, FCPS (Paed. Surg), FICS, Department of Paediatric Surgery, Ibn Sina Specialized Surgical Hospital, Al-Sabah Health Region, Safat, Kuwait, Kuwait 13115, Kuwait (e-mail: dr_yousufaziz@yahoo.com).

Eur J Pediatr Surg Rep 2017;5:e29-e31.
Abstract
Keywords
- Waugh's syndrome
- intussusception
- intestinal malrotation
- children

Waugh's syndrome (WS) is the rare association of intussusception and intestinal malrotation. We present two such cases in a 5-month-old infant and a 9-year-old girl. Both patients presented with intussusception and were diagnosed with malrotation during workup and surgical exploration. We conclude that the possibility of intestinal malrotation must be taken into consideration while managing children with intussusception.
\end{abstract}

\section{New Insights and the Importance for the Pediatric Surgeon}

The association of intestinal malrotation with intussusception (Waugh's Syndrome) should be kept in mind while managing intussusception especially non-surgically. A missed diagnosis of Waugh' syndrome may result in recurrent intussuception and sequel associated with intestinal malrotation increasing morbidity and mortality.

\section{Introduction}

Intussusception is one of the common causes of intestinal obstruction in pediatric population and is mostly (81\%) idiopathic. ${ }^{1}$ Secondary intussusception occurs due to a variety of etiological factors such as Meckel's diverticulum, hypertrophied Peyer's patches, congenital bands, and intestinal lymphomas. ${ }^{1}$ The rare association of intestinal malrotation in conjunction with intussusception was first described by George E Waugh in 1911. It was termed Waugh's syndrome (WS) by Brereton et al after a prospective study confirmed this rare association. ${ }^{2} \mathrm{~A}$ less than 100 cases have been described so far. ${ }^{2,3}$ Herein, we present two such cases managed successfully.

\section{Case Presentation}

Case 1: A 5-month-old female patient weighing $5.2 \mathrm{~kg}$ presented with fever, reluctance to feeds, and excessive crying for

received

June 5, 2017

accepted

June 10, 2017

\author{
DOI https://doi.org/ \\ 10.1055/s-0037-1604264. \\ ISSN 2194-7619.
}

1 day. She had no diarrhea or rectal bleeding. At the age of 3 months, she had an upper gastrointestinal (GI) contrast study done for a brief episode of bilious vomiting. Malrotation was suspected as duodenojejunal (DJ) junction was at the midline and small bowel loops were on right side of abdomen. Since the child improved clinically at that time, a period of observation with repeat upper GI contrast was suggested.

On examination, she had stable vital signs with some dehydration. The abdomen was distended without any palpable abdominal mass and the laboratory results were within normal range. She had an abdominal ultrasound (US) done showing the classic "doughnut sign" of intussusception (-Fig. 1). The patient was subjected to contrast enema reduction, which failed to identify and confirm the reduced intussusception because of distended, contrast-filled bowel loops; hence, she was taken for explorative laparoscopy. An ileoileal intussusception was seen, which was easily reduced. After reduction, the bowel was inspected and intestinal

\footnotetext{
(c) 2017 Georg Thieme Verlag KG Stuttgart · New York
}

\section{License terms}

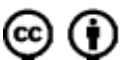




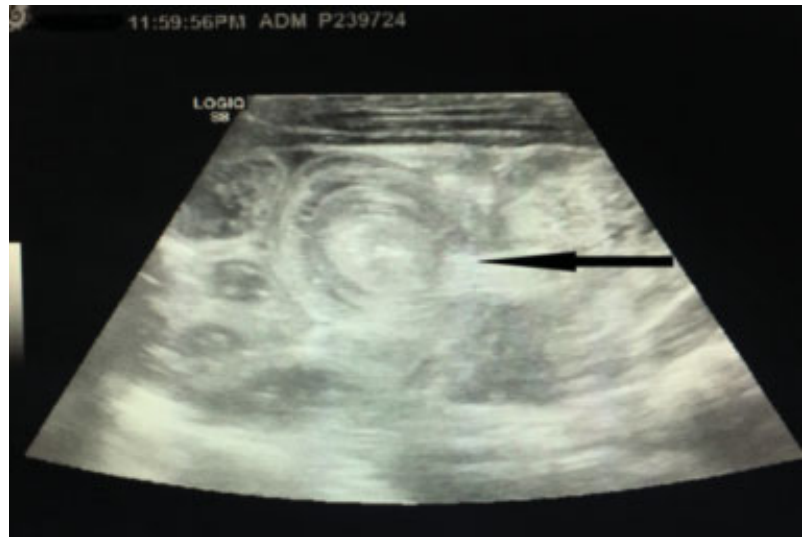

Fig. 1 Case 1: Initial abdominal ultrasound image showing classic "doughnut sign" of intussusception (arrow).

malrotation was suspected. The cecum was located in the midabdomen and most of the small bowel was on the right side. The DJ junction could not be traced due to massively distended small bowel loops; therefore, a minilaparotomy was performed by enlarging the umbilical port site incision. Classical malrotation was confirmed and a standard Ladd's procedure was performed including appendectomy. The postoperative course was uneventful.

Case 2: A 9-year-old female patient weighing $31 \mathrm{~kg}$ was referred with colicky abdominal pain and three episodes of nonbilious vomiting for 1 day. There was no significant past history and the clinical examination was unremarkable. She had a computed tomography (CT) abdomen already done at the office of a primary care physician that showed distal ileal intussusception. We proceeded with an US abdomen that confirmed the initial diagnosis ( - Fig. 2), and a trial of contrast enema reduction was given. The large bowel was noticed on left side of the abdomen with free-lying cecum and the small bowel on right side with free retrograde filling for long segment; the intussusception was not visualized (-Fig. 3). At this stage, intestinal malrotation was taken into consideration. A repeat US abdomen did not show intussusception, and a reversed relation between superior mesenteric artery (SMA) and superior mesenteric vein (SMV) was noticed on Doppler US ( - Fig. 4). An upper GI contrast study showed the DJ junction at the midline and small bowel on right side of the abdomen, suggestive of malrotation.

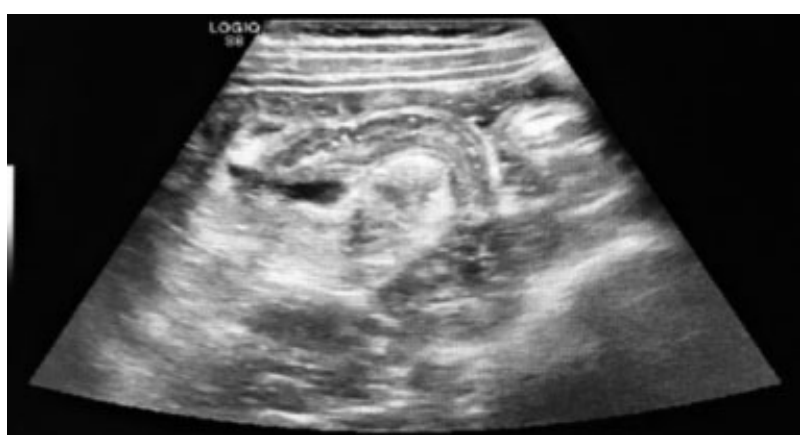

Fig. 2 Case 2: Initial abdominal ultrasound image (longitudinal view) showing telescoping of bowel into the bowel.

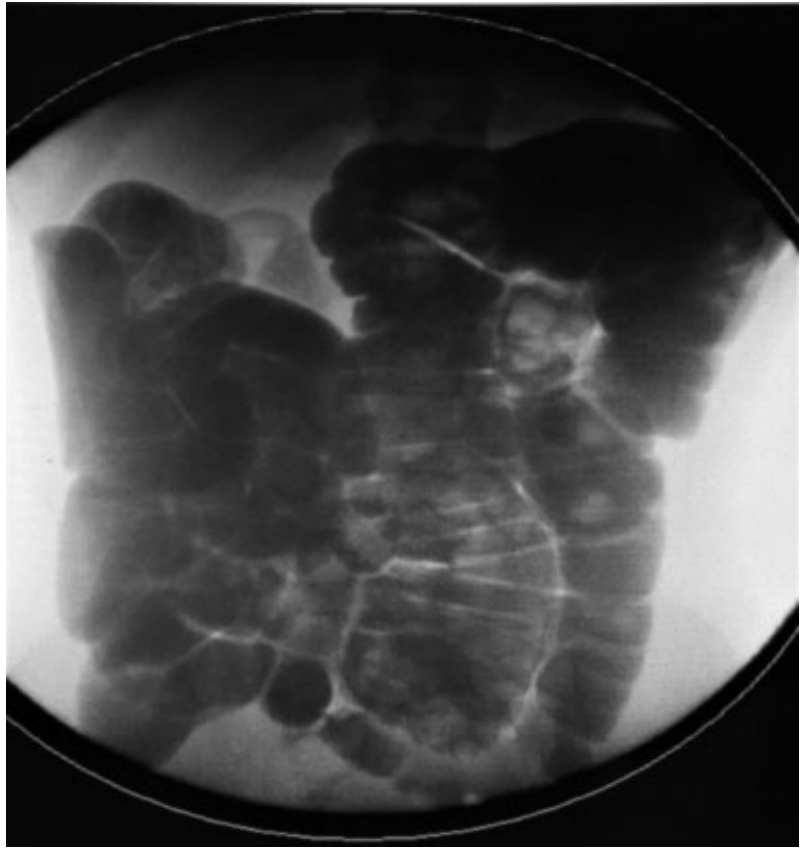

Fig. 3 Case 2: Lower GI contrast study done after US abdomen showing large bowel on the left side and small bowel on the right side of the abdomen. GI, gastrointestinal tract; US, ultrasound.

Therefore, the girl underwent an elective exploratory laparotomy through a right supraumbilical transverse incision. Typical malrotation was found and a standard Ladd's procedure including appendectomy was performed. She had uneventful postoperative course.

\section{Discussion}

The frequency of WS, intussusception in association with malrotation, is not known. In a prospective study, Brereton et al reported atypically positioned DJ junction in 40\% of patients with intussusception in whom the position of DJ junction was determined. ${ }^{4}$ However, the actual incidence of WS may be higher than reported. ${ }^{2,3,5}$ Poorly fixed ascending and descending colon to the retroperitoneum, anomalous

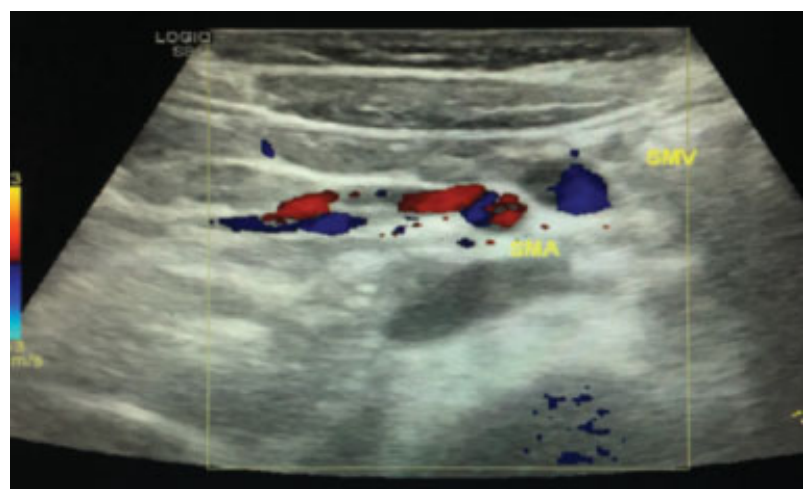

Fig. 4 Case 2: Doppler US image showing reversed relation of SMA and SMV done after the lower GI contrast study was suspicious of malrotation. $\mathrm{Gl}$, gastrointestinal tract; SMA, superior mesenteric artery; SMV, superior mesenteric vein; US, ultrasound. 
rotation, and fixation of the ileocaecal mesentery have been hypothesized as the possible predisposing factors to intussusception in patients with WS. ${ }^{2,5,6}$ Similar features of malrotation were seen at exploration in both our patients.

The age at presentation in WS is highly variable and ranges from 13 days to 17 years. ${ }^{6}$ However, there are reports of WS in a preterm and a 56 -year-old adult. ${ }^{2,7}$ WS clinically presents with classical acute intussusception or vague general and gastrointestinal symptoms; the accompanying malrotation is usually known later on during workup and/or surgical exploration. ${ }^{2,6}$ Both of our patients presented with an acute intussusception, and distinctive features of WS were absent on presentation.

US of abdomen is a dependable diagnostic modality in a case of intussusception with a high sensitivity and specificity. ${ }^{8}$ WS is suspected if the radiologist observes an atypical configuration of the SMA and SMV in a case with intussusception; upper GI contrast study may then be performed to confirm the malrotation. ${ }^{2}$ Following an abdominal US for intussusception, contrast enema reduction can give a clue to the associated intestinal malrotation by localizing the position of cecum, which may be abnormal in 80 to $87 \%$ of surgically proven cases of malrotation. ${ }^{9}$ In our first case, abdominal US confirmed intussusception, but contrast enema was ineffective due to massively distended and overlapping bowel loops. In the second case, intussusception was not visualized on US presumably because it had spontaneously reduced. As an inverted relationship of SMA and SMV was suggested, the patient received an upper GI contrast study to confirm the diagnosis.

Nonsurgical management of intussusception has a significant failure rate if associated malrotation is present. ${ }^{2}$ With a successful enema reduction of intussusception, the diagnosis of WS may remain obscure. It may in turn predispose a patient to recurrent intussusception. ${ }^{2,5}$ Most of the reported cases of WS were managed by open surgery and only one case has been performed laparoscopically. ${ }^{2,5,6}$ In our first case, intussusception was successfully reduced laparoscopically but we could not proceed further because of significant bowel dilatation and inability to define the anatomy clearly.

In conclusion, WS should be kept in mind when managing idiopathic intussusception and in case of additional signs, the clinical workup should include studies to rule out malrotation. $^{10}$

\section{Conflict of Interest}

None.

\section{References}

1 Singh AP, Jangid M, Ansari JS,et al. Rare case of intussusception with malrotation and Meckel's diverticulum. JCR 2014; 4:338-340

2 Al-Momani H. Waugh syndrome: a report of 7 patients and review of the published reports. Ann Saudi Med 2014;34(06): 527-531

3 Baltazar G, Sahyoun C, Sime J, Bitar M, Bitar J, Rao AC. Discovery of a case of Waugh's syndrome during a mission to Haiti. Int J Surg Case Rep 2012;3(01):22-24

4 Brereton RJ, Taylor B, Hall CM. Intussusception and intestinal malrotation in infants: Waugh's syndrome. Br J Surg 1986;73(01): 55-57

5 Behera CR, Mohanty SK. Waugh's Syndrome: blessing in disguise. J Clin Diagn Res 2014;8(10):ND26-ND27

6 Zavras N, Tsilikas K, Vaos G. Chronic intussusception associated with malrotation in a child: avariation of Waugh's syndrome? Case Rep Surg 2016;2016:5638451

7 Saxena R, Puri A, Pinnamaneni R. Waugh syndrome in preterm infant: diagnostic clues. Pediatr Neonatol 2015;56(03):203-204

8 Henderson AA, Anupindi SA, Servaes S, et al. Comparison of 2-view abdominal radiographs with ultrasound in children with suspected intussusception. Pediatr Emerg Care 2013; 29(02):145-150

9 Tackett JJ, Muise ED, Cowles RA. Malrotation: current strategies navigating the radiologic diagnosis of a surgical emergency. World J Radiol 2014;6(09):730-736

10 Gil-Vargas M, Sol-Meléndez AK, Miguel-Sardaneta ML. Is intestinal malrotation the cause of intussusception? Waugh's syndrome, a case report [Article in Spanish]. Cir Cir 2016; $84(03): 250-252$ 